\title{
Structural and functional brain connectivity in presymptomatic familial frontotemporal dementia
}

Elise G.P. Dopper

Serge A.R.B. Rombouts,

$\mathrm{PhD}$

Lize C. Jiskoot, MSc

Tom den Heijer, MD,

$\mathrm{PhD}$

J. Roos A. de Graaf

Inge de Koning, $\mathrm{PhD}$

Anke R. Hammerschlag,

MSc

Harro Seelaar, $\mathrm{PhD}$

William W. Seeley, MD, $\mathrm{PhD}$

Ilya M. Veer, MSc

Mark A. van Buchem,

$\mathrm{MD}, \mathrm{PhD}$

Patrizia Rizzu, PhD

John C. van Swieten,

$\mathrm{MD}, \mathrm{PhD}$

Correspondence to

Dr. van Swieten:

j.c.vanswieten@erasmusmc.nl

Supplemental data at www.neurology.org

\section{ABSTRACT}

Objective: We aimed to investigate whether cognitive deficits and structural and functional connectivity changes can be detected before symptom onset in a large cohort of carriers of microtubuleassociated protein tau and progranulin mutations.

Methods: In this case-control study, 75 healthy individuals (aged $20-70$ years) with $50 \%$ risk for frontotemporal dementia (FTD) underwent DNA screening, neuropsychological assessment, and structural and functional MRI. We used voxel-based morphometry and tract-based spatial statistics for voxelwise analyses of gray matter volume and diffusion tensor imaging measures. Using resting-state $\mathrm{fMRI}$ scans, we assessed whole-brain functional connectivity to frontoinsula, anterior midcingulate cortex (aMCC), and posterior cingulate cortex.

Results: Although carriers $(n=37)$ and noncarriers $(n=38)$ had similar neuropsychological performance, worse performance on Stroop III, Ekman faces, and Happé cartoons correlated with higher age in carriers, but not controls. Reduced fractional anisotropy and increased radial diffusivity throughout frontotemporal white matter tracts were found in carriers and correlated with higher age. Reductions in functional aMCC connectivity were found in carriers compared with controls, and connectivity between frontoinsula and aMCC seeds and several brain regions significantly decreased with higher age in carriers but not controls. We found no significant differences or age correlations in posterior cingulate cortex connectivity. No differences in regional gray matter volume were found.

Conclusions: This study convincingly demonstrates that alterations in structural and functional connectivity develop before the first symptoms of FTD arise. These findings suggest that diffusion tensor imaging and resting-state fMRI may have the potential to become sensitive biomarkers for early FTD in future clinical trials. Neurology ${ }^{\circledR}$ 2013;80:814-823

\section{GLOSSARY}

$\mathbf{A C C}=$ anterior cingulate cortex; aMCC = anterior midcingulate cortex; $\mathbf{D A}=$ axial diffusivity; $\mathbf{D M N}=$ default mode network; $\mathbf{D R}=$ radial diffusivity; $\mathbf{D T I}=$ diffusion tensor imaging; $\mathbf{F A}=$ fractional anisotropy; $\mathbf{F T D}=$ frontotemporal dementia; $\mathbf{G R N}=$ progranulin; $\mathbf{M A P T}=$ microtubule-associated protein tau; $\mathbf{M D}=$ mean diffusivity; $\mathbf{M N I}=$ Montreal Neurological Institute; PCC = posterior cingulate cortex; RAVLT = Rey Auditory Verbal Learning Test; SN = salience network; TMT = Trail Making Test; $\mathbf{V B M}=$ voxel-based morphometry; $\mathbf{W M}=$ white matter.

Frontotemporal dementia (FTD) is characterized by behavioral and language disorders, executive dysfunction, and impaired social cognition. ${ }^{1-3}$ The frontoinsula, anterior cingulate cortex (ACC), and anterior midcingulate cortex (aMCC) are among the first affected brain regions in FTD. ${ }^{4,5}$ However, in early disease, atrophy may be subtle or difficult to detect. ${ }^{6,7}$ With current research focusing on potential disease-modifying treatments, the identification of appropriate biomarkers to detect early FTD and track disease progression is crucial. ${ }^{8,9}$ Microtubule-associated protein tau $(M A P T)$, progranulin $(G R N)$, and C9orf72 mutations are the major genetic causes of autosomal

\footnotetext{
From the Departments of Neurology (E.G.P.D., L.C.J., T.d.H., H.S., J.C.v.S.), Epidemiology (T.d.H.), and Neuropsychology (J.R.A.d.G., I.d.K.), Erasmus Medical Center, Rotterdam; Department of Radiology (E.G.P.D., S.A.R.B.R., I.M.V., M.A.v.B.), Leiden University Medical Center, Leiden; Departments of Neurology (E.G.P.D.) and Clinical Genetics (A.R.H., P.R., J.C.v.S.), VU Medical Center, Amsterdam; Leiden Institute for Brain and Cognition (S.A.R.B.R., I.M.V., M.A.v.B.) and Institute of Psychology (S.A.R.B.R., I.M.V.), Leiden University, Leiden; Department of Neurology (T.d.H.), Sint Franciscus Gasthuis, Rotterdam, the Netherlands; and Department of Neurology (W.W.S.) and Memory and Aging Center (W.W.S.), University of California, San Francisco, CA.

Go to Neurology.org for full disclosures. Funding information and disclosures deemed relevant by the authors, if any, are provided at the end of the article.
} 
dominant FTD. ${ }^{10-12}$ Asymptomatic carriers of these mutations provide the ideal study population to investigate the first alterations in FTD. ${ }^{13}$

Several diffusion tensor imaging (DTI) studies in patients with FTD have reported reduced white matter (WM) integrity, most prominent in the frontotemporal cortex, ${ }^{14-20}$ corresponding to WM pathology. ${ }^{21} \mathrm{~A}$ small study in presymptomatic $G R N$ carriers indicated reduced integrity of the left uncinate and inferior frontooccipital fasciculus. ${ }^{22}$

Resting-state fMRI studies in FTD demonstrated reduced functional connectivity within the salience network (SN), which is involved in emotional processing, and is anchored by the frontoinsula, ACC, and aMCC, spatially corresponding to the specific atrophy pattern in FTD. ${ }^{7,23-25}$ Conflicting results were found in 2 small series of presymptomatic MAPT and GRN carriers. ${ }^{6,7}$

In the current study of a large cohort of presymptomatic MAPT and GRN carriers, we investigated whether cognitive and structural or functional imaging changes occur before symptom onset.

METHODS Subjects. From December 2009 through March 2011, we recruited participants for this case-control study from a pool of 160 healthy first-degree relatives (aged 20-70 years) of patients with FTD due to a $G R N$ or $M A P T$ mutation. We defined subjects as asymptomatic when participant and spouse denied cognitive or behavioral disturbances. We contacted the first 105 subjects, of whom 86 were willing to participate. Reasons for nonparticipation were nonrelated illness, lack of motivation, claustrophobia, and disinterest in disease confrontation. We excluded subjects with MRI contraindications $(n=4)$, history of drug abuse $(\mathrm{n}=2)$, and neurologic or psychiatric disorders $(\mathrm{n}=2)$. DNA of the remaining 78 participants was screened for $G R N$ and MAPT mutations, as previously described. ${ }^{12}$ Family members without a mutation constituted the control group. Researchers remained blinded to the genetic status of participants. Participants underwent neuropsychological assessment, structural MRI, DTI, and resting-state fMRI. We excluded 3 individuals from analyses because of a cerebellar cyst $(n=1)$ or technical failure during scanning $(\mathrm{n}=2)$, and 1 additional participant from DTI analyses because of motion artifacts. For all measures, we performed both between-group comparisons and age correlation analyses, to investigate whether alterations arise as carriers approach their estimated onset age.

Standard protocol approvals, registrations, and patient consents. All participants provided written informed consent. The local ethics committee approved the study.

Neuropsychological assessment. We screened all subjects with the Mini-Mental State Examination, ${ }^{\mathrm{el}}$ Beck Depression Inventory, ${ }^{\mathrm{e}}$ and State-Trait Anxiety Inventory. ${ }^{\mathrm{e}}$ The neuropsychological test battery included the Dutch version of the Rey Auditory Verbal Learning Test (RAVLT) ${ }^{\mathrm{e}}$; stories of the Rivermead Behavioral Memory Test ${ }^{\mathrm{es}}$; Visual Association Test ${ }^{\mathrm{ec}}$; Wechsler Adult Intelligence Scale III subtests digit span, proverbs, similarities, and block design ${ }^{\mathrm{e} 7, \mathrm{e} 8}$; Trail Making Test (TMT) ${ }^{\mathrm{e} 9}$; Stroop color-word test $^{\mathrm{e}^{10}}$; categorical and letter fluency ${ }^{\mathrm{e} 11}$; modified Wisconsin Card Sorting Test ${ }^{\mathrm{e} 2}$; Letter Digit Substitution Test ${ }^{\mathrm{e} 13}$; Boston Naming Test $^{\mathrm{e} 14}$; Semantic Association Test ${ }^{\mathrm{e} 15}$; ScreeLing ${ }^{\mathrm{e} 16}$; clock drawing (Royall) $^{\mathrm{e} 17}$; Cube Copying ${ }^{\mathrm{e1}}$; and the social cognition tests Ekman faces, ${ }^{\mathrm{e} 19}$ Happé cartoons, ${ }^{\text {e20 }}$ and short Faux Pas. ${ }^{\text {e21 }}$

Image acquisition and analyses. We acquired whole-brain anatomical, DTI, and resting-state fMRI scans on a Philips 3.0-T Achieva MRI scanner (Philips Medical Systems, Best, the Netherlands) using an 8-channel SENSE head coil (see appendix e-1 on the Neurology ${ }^{\circledR}$ Web site at www.neurology.org). We used FSL (FMRIB's Software Library, www.fmrib.ox.ac.uk) for all imaging analyses. ${ }^{26}$

We used FSL-VBM, a voxel-based morphometry style analysis giving individual images of gray matter concentration, for T1-weighted scans (see appendix e-1). ${ }^{\text {c22-e29 }}$ We performed permutation-based testing using 5,000 permutations, applying a 2-sample $t$ test model with confound regressors for age and gender, to compare regional gray matter and to investigate correlations with age (see www.fmrib.ox.ac.uk/fsl/randomise/index). We set the statistical threshold at $p<0.05$, corrected for multiple comparisons using threshold-free cluster enhancement. ${ }^{228}$

For voxelwise comparisons of multiple DTI measures, including fractional anisotropy (FA), mean diffusivity (MD), axial diffusivity (DA), and radial diffusivity (DR), we used tract-based

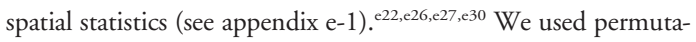
tion testing with 5,000 permutations, applying a 2-sample $t$ test, with age and gender as confound regressors, for between-group and age correlation analyses. We thresholded the resulting statistical maps at $p<0.05$, corrected for multiple comparisons using threshold-free cluster enhancement. ${ }^{\text {e28 }}$

For resting-state fMRI data, we used a seed-based approach in the FMRI Expert Analysis Tool. After standard preprocessing (see appendix e-1), ${ }^{e 22, e 24,225}$ we conducted within-subject analyses to determine whole-brain regional connectivity with 3 bilateral seed regions of interest: left and right frontoinsula (36-voxel clusters around Montreal Neurological Institute [MNI] coordinates $\mathrm{x}= \pm 38, \mathrm{y}=26, \mathrm{z}=-10)$ and aMCC (4-mm spheres around $\mathrm{x}= \pm 5, y=19, \mathrm{z}=28)$ to define the SN and left and right posterior cingulate cortex (PCC) (4-mm spheres around $\mathrm{x}= \pm 2$, $\mathrm{y}=-51, \mathrm{z}=27)$ to define the default mode network $(\mathrm{DMN}){ }^{24,27,28}$ We extracted mean time series for each seed for each subject in native space by applying the inverse transform from MNI space to fMRI. For each individual, we performed time-series statistical analyses per seed with local autocorrelation correction using the General Linear Model, which included time series for WM, CSF, and the global signal, and 6 motion parameters as confound regressors. We acquired parameter estimates for each regressor. Contrasts of interest were the left and right seed separately and the left and right seeds together, for all regions of interest (frontoinsula, aMCC, and PCC). We transformed subject-level contrast images and corresponding images of variance to MNI space using the combined transformation matrix, for group analysis. For group analyses, we used a 2 -sample $t$ test, with age, gender, and voxelwise gray matter volume included as additional regressors to investigate between-group differences and age correlations. We thresholded $z$-statistic images using clusters determined by an initial cluster-forming threshold $z>2.3$ and a corrected cluster significance threshold of $p<0.05 .{ }^{\text {e29 }}$ Age correlation analyses were Bonferroni-corrected for multiple testing. 
Table 1 Demographic features and neuropsychological performance ${ }^{a}$

(Maximum scores)
Age, y
Females
GRN mutation ${ }^{c}$
Level of education $^{d}$
MMSE score (30)
BDI (63)

\section{STAI DY1 (80)}

STAI DY2 (80)

Attention and executive functions

Digit span (30)
Proverbs (8)
Similarities (33)
TMT A
TMT B
Stroop I
Stroop II
Stroop III
Categorical fluency
Letter fluency
WCST concepts (6)
LDST $^{f}$
Memory

Orientation (10)

\section{RAVLT recall (15)}

RAVLT recognition (30)

RBMT immediate (42)

RBMT delayed (42)

VAT (12)

Language

BNT (60)

\section{SAT verbal (30)}

SAT nonverbal (30)

ScreeLing (72)

Visuospatial

Royall clock (14)

Cube Copying (8)

Block design (68)

Social cognition

Ekman faces (60)
Total study group ( $n=75$ ): Age range 22.2-68.6 y

$\begin{array}{lll}\text { Controls }(\mathbf{n}=38) & \begin{array}{l}\text { Mutation carriers } \\ (\mathbf{n}=37)\end{array} & p \text { Value }^{\mathrm{b}} \\ 50.2(11.5) & 51.0(9.6) & 0.744 \\ 53 \% & 57 \% & 0.720 \\ - & 76 \% & - \\ 5.1(1.1) & 5.5(1.1) & 0.087 \\ 29.1(1.3) & 29.2(1.5) & 0.742 \\ 3.2(4.1) & 3.9(4.6) & 0.214 \\ 32.4(5.7) & 35.3(10.3) & 0.054 \\ 32.1(7.0) & 34.5(10.8) & 0.063\end{array}$

$15.0(3.5)$

15.6 (3.6)

0.914

6.1 (1.7)

$6.6(1.4)$

0.345

$24.6(4.6)$

31.4 (14.2)

$66.2(30.6)$

$46.4(7.7)$

58.7 (10.3)

$92.6(21.8)$

$42.2(8.4)$

32.5 (9.5)

$5.5(1.0)$

$34.3(6.8)$

$9.9(0.3)$

43.8 (11.0)

8.8 (3.4)

$28.6(2.1)$

$26.0(5.0)$

0.772

31.1 (11.8)

0.675

72.7 (39.8)

0.119

45.1 (8.9)

0.730

59.1 (12.7) $\quad 0.600$

94.7 (25.9) $\quad 0.390$

40.8 (10.2) $\quad 0.242$

$36.7(12.7) \quad 0.380$

$5.8(0.5) \quad 0.082$

33.4 (7.2) $\quad 0.118$

$9.8(0.6) \quad 0.180$

$44.7(9.4) \quad 0.835$

$8.9(3.4) \quad 0.637$

28.9 (1.5) $\quad 0.847$

$20.6(6.0) \quad 0.692$

$16.9(6.1) \quad 0.970$

11.4 (1.2) $\quad 0.121$

$11.8(0.6)$

$53.5(4.2) \quad 54.6(4.2) \quad 0.610$

$27.8(1.3) \quad 27.5(1.9) \quad 0.470$

$28.7(1.4) \quad 29.1(1.0) \quad 0.312$

$\begin{array}{lll}70.8(1.5) & 71.4(0.7) & 0.078\end{array}$

$12.3(1.6) \quad 12.5(1.3) \quad 0.737$

$7.8(0.5) \quad 7.9(0.5) \quad 0.694$

$35.3(13.1) \quad 35.3(15.7) \quad 0.658$

$46.8(5.9)$
We re-ran all analyses with $\mathrm{SN}$ and DMN templates (see appendix e-1) $)^{\text {e31,e32 }}$ as prethreshold masks to determine which differences were within-network alterations.

Statistical analyses. We performed statistical analyses in SPSS 17.0 for Windows (SPSS, Chicago, IL). We analyzed demographic features using independent samples $t$ tests and Pearson $\chi^{2}$ tests, and neuropsychological data using analyses of covariance controlling for age, gender, and education. We applied a significance level of $p<0.05$ (2-tailed) across all analyses.

RESULTS Demographic features. Genetic screening revealed 37 mutation carriers (GRN mutation in 28, $M A P T$ in 9) and 38 noncarriers. Age, gender, and education level, ${ }^{29}$ and Mini-Mental State Examination, Beck Depression Inventory, and State-Trait Anxiety Inventory scores of carriers and controls were similar (table 1). The current age of MAPT carriers (41.7 \pm 8.8 years) was $7.4 \pm 7.8$ years younger than the mean onset age in their families; the current age of $G R N$ carriers ( $54.0 \pm 7.8$ years) was $8.6 \pm 7.4$ years younger than their families' mean onset age $(p=0.690)$.

Neuropsychological assessment. Carriers and controls performed similarly on all cognitive tests (table 1). Worse performances on Stroop III, Ekman faces, and Happé cartoons significantly correlated with higher age in carriers, but not controls (table e-1, figure 1A). Conversely, scores on Cube Copying and proverbs increased with higher age in carriers, with a trend for similar age effects on proverbs performances in controls. Performances on TMT A and RAVLT total and recall decreased with higher age in both groups, whereas block design and Faux Pas scores decreased only in controls. The age effects in Cube Copying and Faux Pas performance are probably a result of ceiling effects, due to the limited range in test scores.

Structural imaging: VBM. VBM analysis revealed no significant differences in regional gray matter volume between controls and carriers. A decline in gray matter volume with higher age was found in widespread regions in both groups, whereas there were no areas showing an increase in gray matter volume in either group.

Structural connectivity: Tract-based spatial statistics. Carriers showed significantly decreased FA in various frontotemporal WM tracts in carriers compared with controls, including right uncinate fasciculus, superior and inferior longitudinal fasciculi, superior corona radiata, external capsule, and anterior and posterior limb of internal capsule, and bilateral forceps minor, anterior corona radiata, anterior thalamic radiation, inferior frontooccipital fasciculus, and the body of the corpus callosum (figure 2A, table e-2). Increases in DR were found in similar, albeit less-extensive, areas. 
Table 1 Continued

\begin{tabular}{llll} 
& \multicolumn{3}{l}{ Total study group $(\mathbf{n}=\mathbf{7 5})$ : Age range 22.2-68.6 y } \\
\cline { 2 - 4 } (Maximum scores) & Controls $(\mathbf{n}=\mathbf{3 8})$ & $\begin{array}{l}\text { Mutation carriers } \\
(\mathbf{n}=37)\end{array}$ & $\mathbf{p}$ Value ${ }^{\mathrm{b}}$ \\
Happé cartoons (36) & $23.7(5.7)$ & $25.3(5.2)$ & 0.325 \\
Faux Pas (10) & $9.0(1.1)$ & $9.1(1.1)$ & 0.684
\end{tabular}

Abbreviations: BDI = Beck Depression Inventory; BNT = Boston Naming Test; $G R N=$ progranulin; LDST = Letter Digit Substitution Test; MAPT = microtubule-associated protein tau; MMSE = Mini-Mental State Examination; RAVLT = Rey Auditory Verbal Learning Test; RBMT $=$ Rivermead Behavioral Memory Test; SAT = Semantic Association Test; STAI = State-Trait Anxiety Inventory (versions DY1 and DY2); TMT = Trail Making Test; VAT = Visual Association Test; WCST $=$ Wisconsin Card Sorting Test.

a Values denote mean (SD) or percentage of subjects. Scores on SAT nonverbal were missing in 3 individuals, and scores on block design in 2.

${ }^{b} p$ Values for demographic features are from independent samples $t$ tests; $p$ values for neuropsychological performances are from analysis of covariance controlling for age, gender, and education.

${ }^{\mathrm{c}}$ Remaining mutation carriers have a MAPT mutation.

${ }^{\mathrm{d}}$ Level of education was determined on a Dutch 7-point scale ranging from 1 (less than elementary school) to 7 (university or technical college). ${ }^{29}$

e Time in seconds.

${ }^{\mathrm{f}}$ Number of correct responses in 1 minute.

In carriers, but not in controls, FA significantly decreased and DR increased with higher age in the above-mentioned WM tracts (figures $1 \mathrm{~B}$ and $2 \mathrm{~B}$, table $\mathrm{e}-2)$. The pattern of these age correlations was more widespread and symmetrical than the mainly right-sided pattern of between-group differences. No group differences or age correlations were found for MD and DA.

Separate analysis of GRN carriers $(\mathrm{n}=27)$ vs controls from their families $(\mathrm{n}=28)$ revealed decreased FA in the right anterior and superior corona radiata, anterior thalamic radiation, superior longitudinal fasciculus, inferior frontooccipital fasciculus, forceps minor, and anterior and posterior limb of internal capsule (figure 3A).
MAPT carriers $(\mathrm{n}=9)$ showed significantly decreased FA and increased MD, DR, and DA compared with controls $(\mathrm{n}=10)$ in widespread frontotemporal WM tracts (figure 3B).

Functional connectivity: Frontoinsula seed. Although no significant differences in frontoinsula connectivity between carriers and controls were found, lower connectivity of the frontoinsula seeds with the pregenual ACC, aMCC, paracingulate gyrus, frontal pole, right superior frontal gyrus and left supramarginal gyrus, angular gyrus, lateral occipital cortex, and parietal opercular cortex correlated significantly with higher age in carriers, but not in controls (figures $1 \mathrm{C}$ and 4A, table e-3). Separate analysis for MAPT and GRN revealed no significant differences. Using the $\mathrm{SN}$ template as prethreshold mask, only the cluster of pregenual ACC, aMCC, paracingulate cortex, and frontal pole remained.

Functional connectivity: aMCC seed. Significantly decreased right aMCC connectivity with the right PCC, precuneus, supramarginal, postcentral, and angular gyri, and superior parietal cortex was found in carriers compared with controls (figure $4 \mathrm{~B}$, table e-3). In carriers and not in controls, advancing age correlated with reduced aMCC seed connectivity with various brain regions, including right hippocampus, amygdala, left lateral occipital cortex, bilateral lingual gyrus, occipital fusiform cortex, thalamus, brainstem, precuneus, and supplementary motor cortex (figure 4C, table e-3). Increased connectivity between left aMCC seed and some posterior regions with advancing age was found in carriers.

Separate analysis of $G R N$ carriers vs their family controls revealed decreased right aMCC connectivity with precuneus, PCC, supramarginal and postcentral

Figure 1 Relationship between age and neuropsychological performance, structural and functional connectivity in mutation carriers

A Neuropsychological assessment

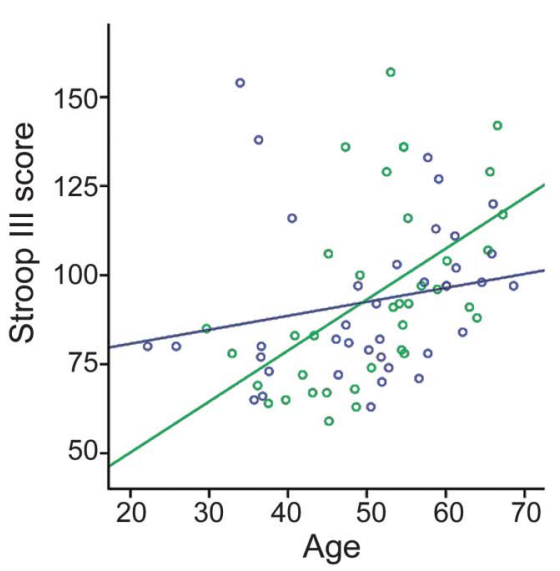

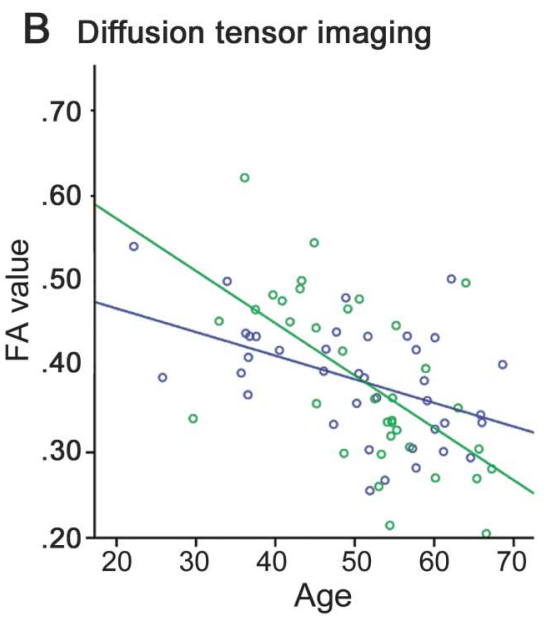

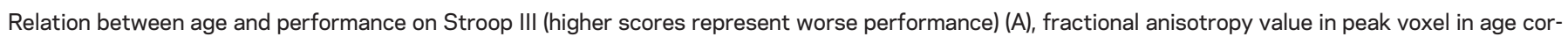

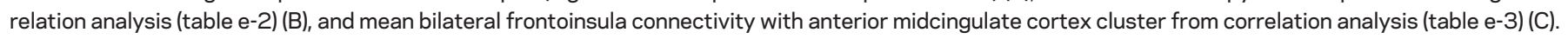




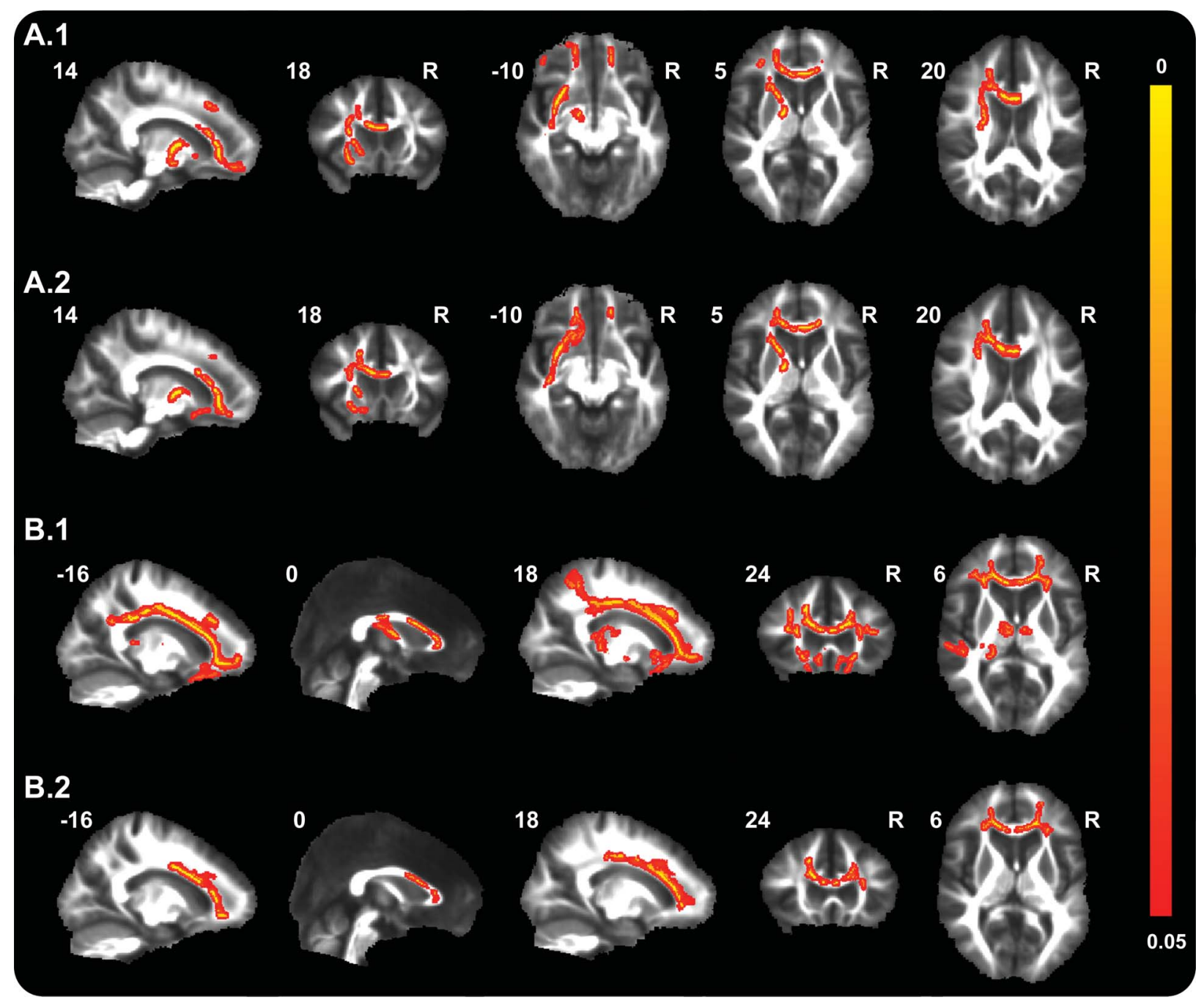

Maps illustrate significant decreases in fractional anisotropy (FA) (A1) and increases in radial diffusivity (DR) (A2) in mutation carriers compared with controls, and significant decreases in FA (B1) and increases in DR (B2) with higher age in carriers. Thresholded statistic images were thickened using tbss_fill in FSL (FMRIB's Software Library) for better visibility. Color bar represents $p$ values.

gyri, and superior parietal cortex. MAPT carriers showed no significant difference compared with controls. Using the SN template as a prethreshold mask, no significant differences between carriers and controls were found in the total group or the MAPT and GRN subgroups.

Functional connectivity: PCC seed. PCC connectivity did not differ between carriers and controls, except for increased out-of-network connectivity with the cerebellum in carriers (table e-3). PCC connectivity was not correlated with age. Separate analyses for $G R N$ and MAPT revealed no differences in PCC connectivity between carriers and controls. Using the PCC template as prethreshold mask, no differences were found.

DISCUSSION This study demonstrates that alterations in structural and functional brain connectivity emerge in FTD mutation carriers before the first symptoms of dementia arise. Our findings have important implications for future clinical trials, suggesting that DTI and resting-state fMRI could possibly be developed into quantitative biomarkers for detecting FTD presymptomatically.

Our finding of a decline in performances on Stroop III, Ekman faces, and Happé cartoons with increasing age in carriers, but not controls, suggests that impairment on these tasks is associated with disease activity before symptom onset. This is further supported by previous observations that social cognition and executive deficits are the earliest neuropsychological characteristics of FTD. ${ }^{1,2,30}$ The executive dysfunction in carriers decades before the estimated disease onset in a previous small study, suggesting a developmental origin of these deficits, ${ }^{31}$ could not be confirmed in the current and another ${ }^{22}$ study, because we found no between-group 

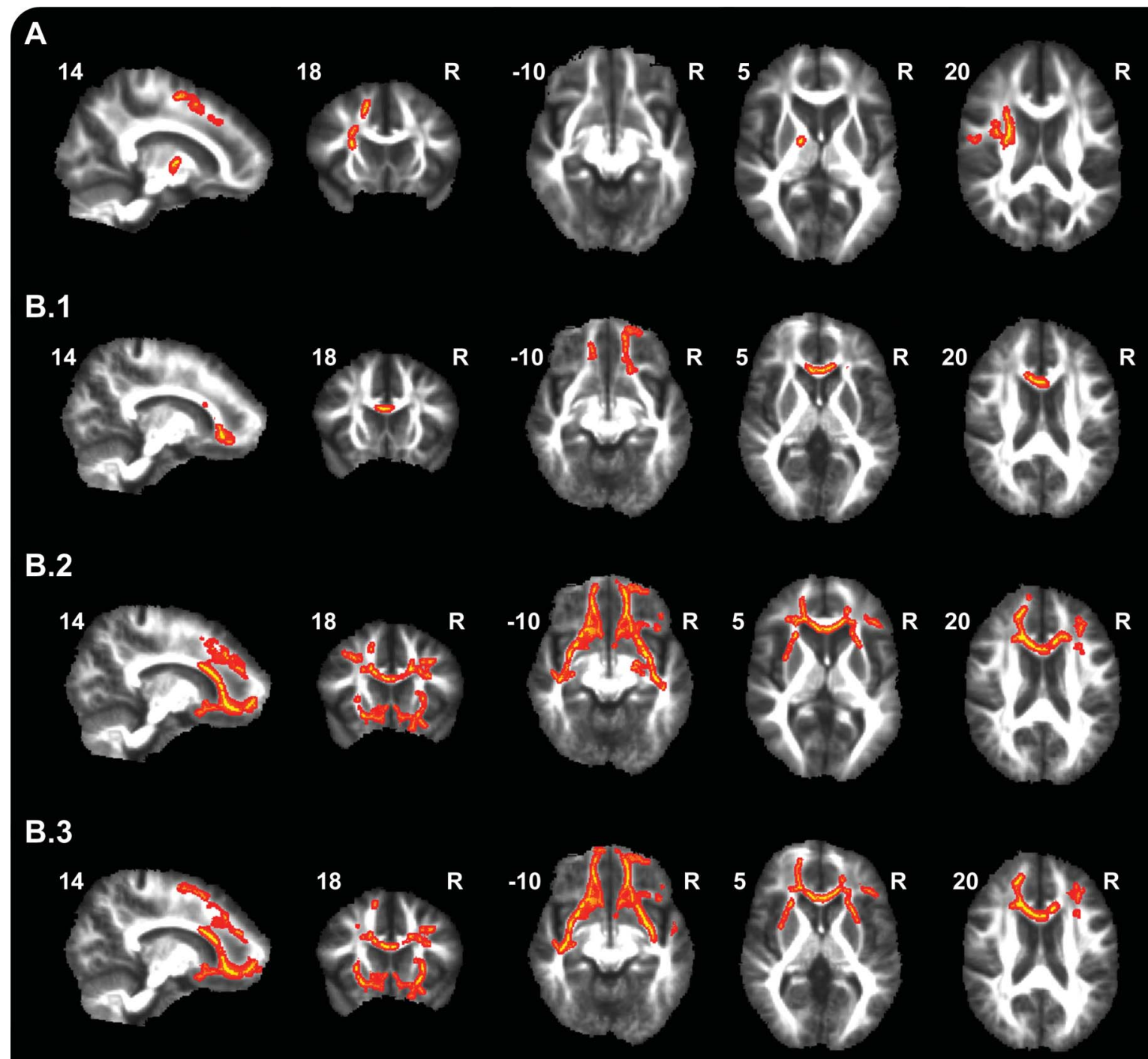

\section{B.4}
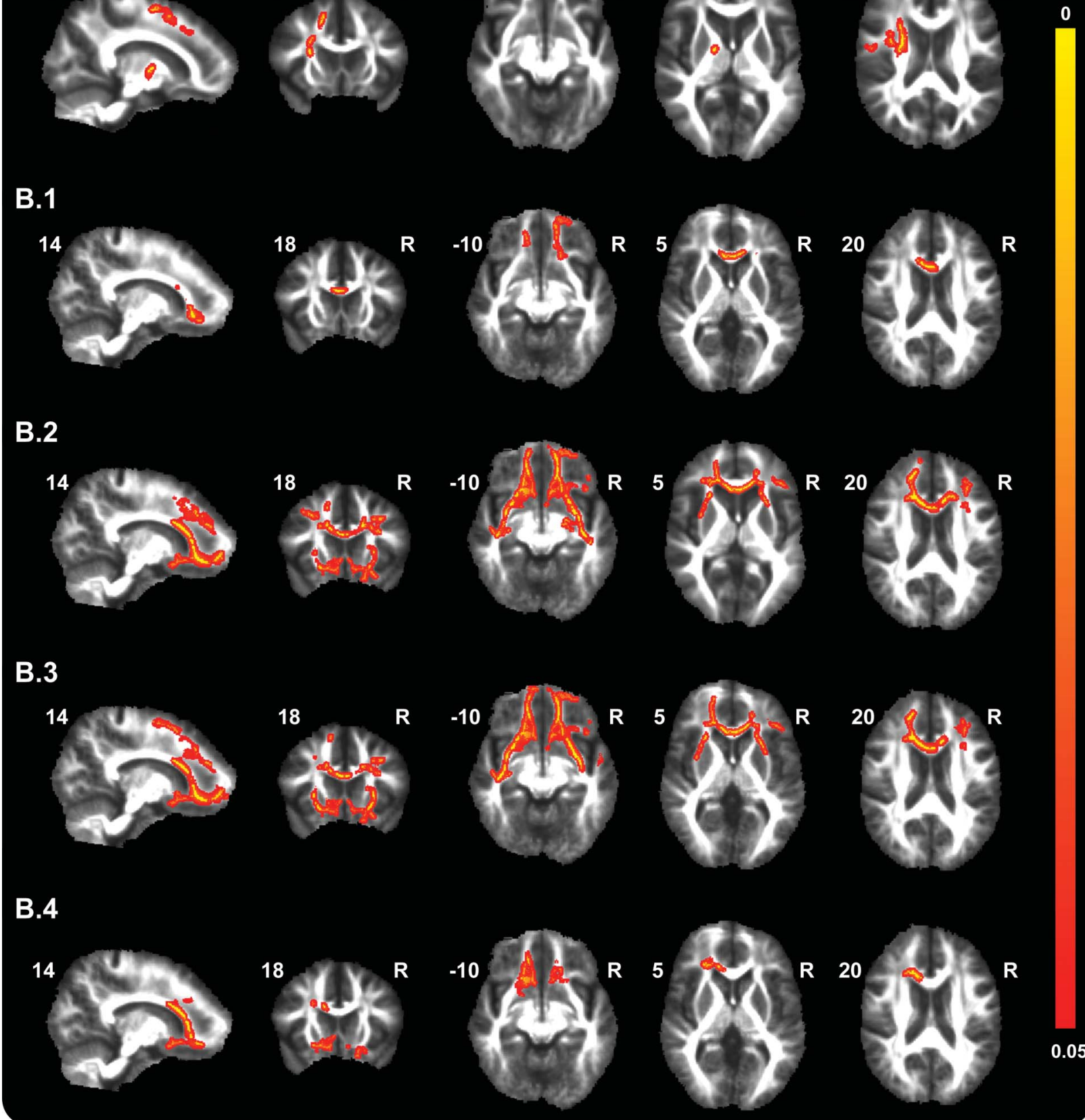

Maps illustrate significant decreases in fractional anisotropy (FA) in GRN mutation carriers compared with controls from their families (A) and significant decreases in FA (B1) and increases in mean diffusivity (B2), radial diffusivity (B3), and axial diffusivity (B4) in MAPT mutation carriers compared with controls from their families. Thresholded statistic images were thickened using tbss_fill in FSL (FMRIB's Software Library) for better visibility. Color bar represents $p$ values.

differences. Decreasing performances on TMT A and RAVLT in carriers and controls probably reflect normal aging effects, whereas increasing scores on proverbs with higher age are probably explained by greater familiarity with proverbs among older participants. Previous studies have shown an age-related decline in block design performance in healthy individuals, as observed in the

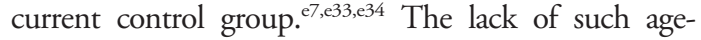
related decline in the mutation carriers is in line with the hypothesis that visuospatial functions are preserved or even enhanced in FTD. ${ }^{25}$

The widespread FA reductions and DR increases in carriers compared with controls are an exciting observation and support the hypothesis that WM 


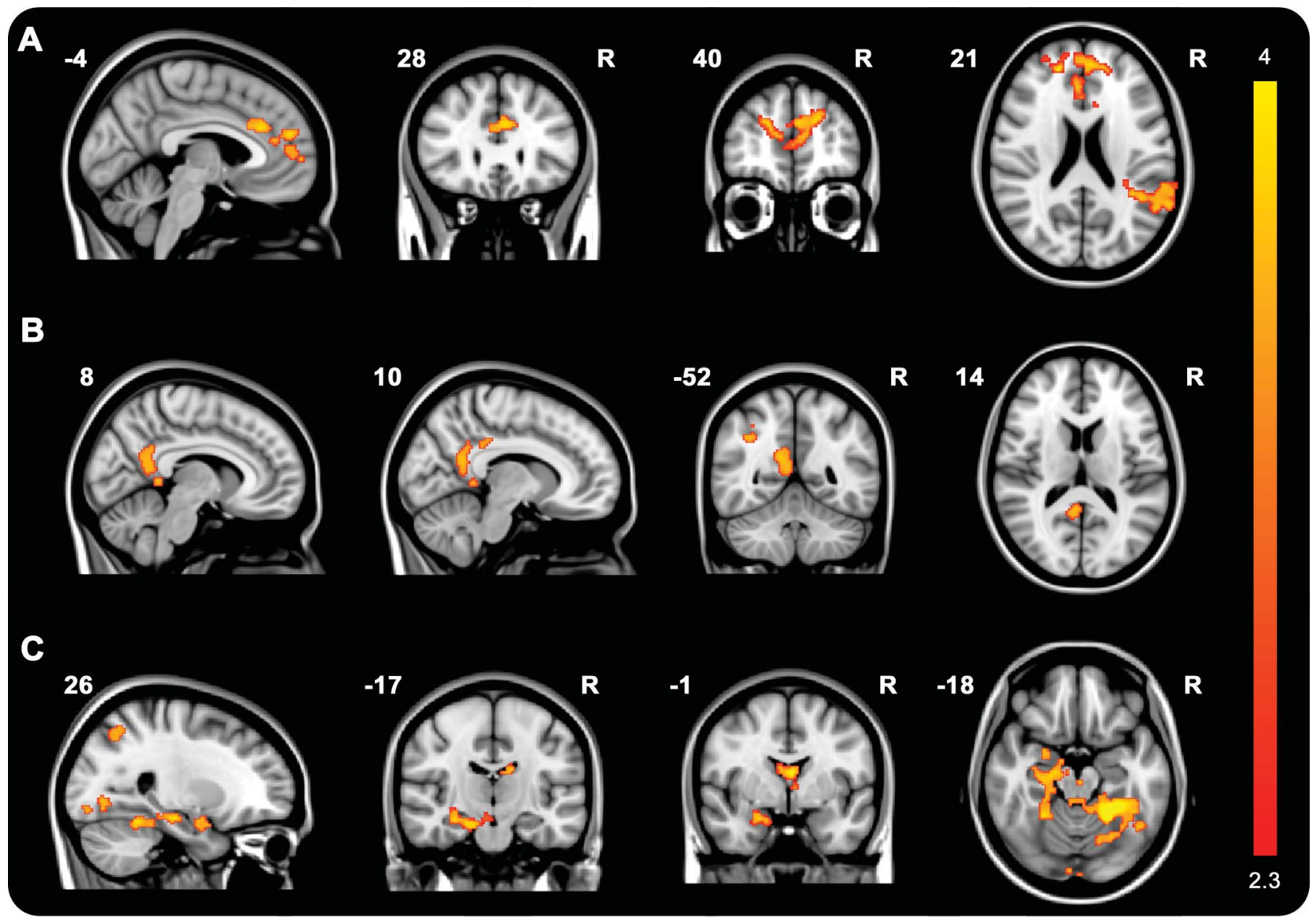

Maps illustrate clusters of significantly decreasing bilateral frontoinsula connectivity in mutation carriers (A) with higher age, significantly decreased aMCC connectivity in mutation carriers compared with controls (B), and decreasing aMCC connectivity with higher age in mutation carriers (C). Color bar represents $z$ scores.

damage is an early feature in the FTD disease process, which is in line with a previous study in presymptomatic GRN carriers. ${ }^{22}$ The distribution of WM damage in the frontotemporal lobes corresponds to that found in the patients with FTD. ${ }^{14-20}$ Reduced integrity of the uncinate fasciculus and anterior corpus callosum is a consistent finding in patients with FTD ${ }^{14,16,32}$ and correlates with disinhibition, ${ }^{32}$ which is an early feature of FTD. ${ }^{1,3}$ The more widespread pattern of reducing WM integrity with higher age suggests that WM damage spreads as carriers are approaching the symptomatic stage of the disease. The lack of significant differences in MD may be explained by the lower sensitivity of this parameter compared with FA in early dementia. ${ }^{33}$ The divergence between DR and DA findings suggests that myelin breakdown precedes axonal damage in FTD. ${ }^{34,35}$ However, this should be interpreted with some caution, as research into the implications of the different diffusion parameters is still in an early stage. ${ }^{33}$

The finding of WM changes in both MAPT and $G R N$ carriers vs controls underlines that WM degeneration is an early feature in FTD, irrespective of the underlying mutation. The symmetrical distribution of WM damage in MAPT carriers vs the asymmetrical pattern in GRN carriers is in line with the previously reported mutation-specific atrophy patterns. ${ }^{36,37}$ The more widespread WM changes in MAPT carriers, with extensive changes in all DTI measures in contrast to the more focal changes in FA only in $G R N$ carriers, may suggest a mutation-specific distribution of WM damage, because the time until estimated disease onset was similar in MAPT and GRN carriers. One previous study investigated WM changes in FTD patients with MAPT and GRN mutations using VBM, and revealed more widespread WM changes and a faster rate of whole-brain atrophy in GRN than $M A P T$ carriers. ${ }^{36}$ This may suggest that the onset of WM degeneration is earlier in MAPT carriers, but that the progression is faster in GRN carriers. However, the current results should be interpreted with caution because of the relatively small MAPT group.

The decreased connectivity of aMCC with posterior regions, including PCC and precuneus, is remarkable, but corresponds to the more generalized 
pattern of atrophy in $G R N$ mutations, ${ }^{36,37}$ and may therefore relate to the high proportion of $G R N$ carriers, which is further supported by similar findings in the separate $G R N$ analysis. The finding of declining connectivity between frontoinsula and aMCC with higher age in carriers but not in controls corroborates findings of SN impairment in patients with FTD, because these regions are central nodes of this network. $^{24,25}$ The SN has an important role in socialemotional processing, and failing connectivity between its central nodes may relate to the impaired social behavior that typically occurs in early FTD. ${ }^{24}$ The hypothesis that decreasing frontoinsula connectivity with higher age in carriers probably reflects the disease process is further supported by previous observations of reducing frontoinsula connectivity within the SN with advancing disease severity in symptomatic FTD. ${ }^{25}$

The increased PCC connectivity with the cerebellum is in line with increased cerebellar activation in taskrelated fMRI in patients with FTD, ${ }^{38}$ and might represent compensation given its role in executive functions. ${ }^{39}$ The increased connectivity within the DMN found in symptomatic FTD probably arises around the time of symptom onset, as this was not found in the current and a previous study in presymptomatic FTD. ${ }^{6,25}$ The reduced DMN connectivity in MAPT carriers in a recent study may be mutation-specific, ${ }^{7}$ but we could not confirm these findings. However, larger studies of MAPT carriers are needed to further elucidate the specific alterations within this group.

The relationship between structural and functional connectivity is still a subject of discussion. Several studies have indicated a general correspondence between the two, but functional connectivity has also been observed between regions without direct structural connections. ${ }^{40}$ Although structural connectivity changes appeared to be more widespread than functional alterations in the current carriers, a direct comparison between these measures remains difficult because of large differences in analytical methods. More studies combining these MRI techniques are needed to further elucidate this relationship.

The observed connectivity changes in the presymptomatic stage of FTD highlight the potential of MRI to detect disease-related changes. The starting point of decrease in functional connectivity may represent an ideal moment to begin future therapeutic interventions. DTI and resting-state fMRI may have the potential to become diagnostic tools in future clinical trials. The wide availability of MRI scanners, lack of exposure to radioactivity, and independence of task performance are major advantages of these techniques for implementation in clinical trials. Correlation of connectivity changes with age indicates that these tools might also be useful in tracking disease progression. Longitudinal studies are needed to explore these possibilities. Moreover, additional work is required to investigate how pharmacologic interventions affect functional connectivity.

Strengths of our study included the large number of participating at-risk individuals, the extensive neuropsychological battery, and the combination of functional and structural imaging. The major limitation was the limited number of MAPT carriers, and larger studies are needed to further investigate early changes in this subgroup. Furthermore, it would be interesting to investigate connectivity alterations in carriers of the recently discovered C9orf72 repeat expansion, and compare these with MAPT and GRN carriers. Finally, cognitive and connectivity changes with increased age are based on cross-sectional data and should be confirmed in follow-up studies.

This study provides evidence that brain pathophysiology is disrupted in FTD years before symptom onset and these alterations in presymptomatic FTD can be measured with DTI and resting-state fMRI. Structural and functional connectivity may have the potential to be developed into sensitive biomarkers in clinical trials in presymptomatic individuals. These measures may also be sensitive to disease progression, a hypothesis we hope to pursue in follow-up studies.

\section{AUTHOR CONTRIBUTIONS}

Elise G.P. Dopper was involved in data collection, analysis of neuropsychological and MRI data, and writing of the manuscript. Serge A.R.B. Rombouts contributed in the design of the resting-state fMRI study, the analysis of MRI data, and writing of the manuscript. Lize C. Jiskoot contributed to the analysis and interpretation of MRI data. Tom den Heijer contributed to the design of the resting-state fMRI study and writing of the manuscript. J. Roos A. de Graaf was involved in neuropsychological data collection, analyzing the data, and the writing process. Inge de Koning contributed in the design of the neuropsychological study, neuropsychological data collection, and the writing process. Anke R. Hammerschlag was involved in data collection regarding genetic status. Harro Seelaar contributed to data collection, interpretation of the data, and was involved in the writing process. William W. Seeley contributed to the design of the restingstate fMRI study, the interpretation of the data, and in writing of the manuscript. Ilya M. Veer was involved in the analysis and interpretation of MRI data, and in writing of the manuscript. Mark A. van Buchem was involved in the design of the study, data collection, and in the writing process. Patrizia Rizzu was involved in data collection regarding genetic status, and in the writing process. John C. van Swieten contributed in the design of the resting-state fMRI study, data collection and interpretation, and in writing of the manuscript.

\section{STUDY FUNDING}

Supported by Dioraphte Foundation grant 09-02-03-00, the Association for Frontotemporal Dementias Research Grant 2009, The Netherlands Organization for Scientific Research grant HCMI 056-13-018, and Netherlands Alzheimer Foundation. S.R. is supported by a grant from The Netherlands Organization for Scientific Research.

\section{DISCLOSURE}

The authors report no disclosures relevant to the manuscript. Go to Neurology.org for full disclosures.

Received August 17, 2012. Accepted in final form November 5, 2012. 


\section{REFERENCES}

1. Neary D, Snowden JS, Gustafson L, et al. Frontotemporal lobar degeneration: a consensus on clinical diagnostic criteria. Neurology 1998;51:1546-1554.

2. Snowden JS, Gibbons ZC, Blackshaw A, et al. Social cognition in frontotemporal dementia and Huntington's disease. Neuropsychologia 2003;41:688-701.

3. Rascovsky K, Hodges JR, Knopman D, et al. Sensitivity of revised diagnostic criteria for the behavioural variant of frontotemporal dementia. Brain 2011;134:2456-2477.

4. Boccardi M, Sabattoli F, Laakso MP, et al. Frontotemporal dementia as a neural system disease. Neurobiol Aging 2005;26:37-44.

5. Seeley WW, Crawford R, Rascovsky K, et al. Frontal paralimbic network atrophy in very mild behavioral variant frontotemporal dementia. Arch Neurol 2008;65:249-255.

6. Borroni B, Alberici A, Cercignani M, et al. Granulin mutation drives brain damage and reorganization from preclinical to symptomatic FTLD. Neurobiol Aging 2012;33:2506-2520.

7. Whitwell JL, Josephs KA, Avula R, et al. Altered functional connectivity in asymptomatic MAPT subjects: a comparison to bvFTD. Neurology 2011;77:866-874.

8. Rogers BS, Lippa CF. A clinical approach to early-onset inheritable dementia. Am J Alzheimers Dis Other Demen 2012;27:154-161.

9. Cenik B, Sephton CF, Dewey CM, et al. Suberoylanilide hydroxamic acid (vorinostat) up-regulates progranulin transcription: rational therapeutic approach to frontotemporal dementia. J Biol Chem 2011;286:1610116108.

10. Dejesus-Hernandez M, Mackenzie IR, Boeve BF, et al. Expanded GGGGCC hexanucleotide repeat in noncoding region of C9ORF72 causes chromosome 9p-linked FTD and ALS. Neuron 2011;72:245-256.

11. Renton AE, Majounie E, Waite A, et al. A hexanucleotide repeat expansion in C9ORF72 is the cause of chromosome 9p21-linked ALS-FTD. Neuron 2011;72:257-268.

12. Seelaar H, Kamphorst W, Rosso SM, et al. Distinct genetic forms of frontotemporal dementia. Neurology 2008;71:1220-1226.

13. Rohrer JD. Structural brain imaging in frontotemporal dementia. Biochim Biophys Acta 2012;1822:325-332.

14. Agosta F, Scola E, Canu E, et al. White matter damage in frontotemporal lobar degeneration spectrum. Cereb Cortex 2012;22:2705-2714.

15. Borroni B, Brambati SM, Agosti C, et al. Evidence of white matter changes on diffusion tensor imaging in frontotemporal dementia. Arch Neurol 2007;64:246-251.

16. Matsuo K, Mizuno T, Yamada K, et al. Cerebral white matter damage in frontotemporal dementia assessed by diffusion tensor tractography. Neuroradiology 2008;50: 605-611.

17. Whitwell JL, Avula R, Senjem ML, et al. Gray and white matter water diffusion in the syndromic variants of frontotemporal dementia. Neurology 2010;74:12791287.

18. Yoshiura T, Mihara F, Koga H, et al. Cerebral white matter degeneration in frontotemporal dementia detected by diffusion-weighted magnetic resonance imaging. Acad Radiol 2006;13:1373-1378.

19. Zhang Y, Schuff N, Du AT, et al. White matter damage in frontotemporal dementia and Alzheimer's disease measured by diffusion MRI. Brain 2009;132:2579-2592.
20. McMillan CT, Brun C, Siddiqui S, et al. White matter imaging contributes to the multimodal diagnosis of frontotemporal lobar degeneration. Neurology 2012;78:17611768.

21. Larsson EM, Englund E, Sjobeck M, Latt J, Brockstedt S. MRI with diffusion tensor imaging post-mortem at $3.0 \mathrm{~T}$ in a patient with frontotemporal dementia. Dement Geriatr Cogn Disord 2004;17:316-319.

22. Borroni B, Alberici A, Premi E, et al. Brain magnetic resonance imaging structural changes in a pedigree of asymptomatic progranulin mutation carriers. Rejuvenation Res 2008;11:585-595.

23. Seeley WW, Crawford RK, Zhou J, Miller BL, Greicius MD. Neurodegenerative diseases target large-scale human brain networks. Neuron 2009;62:42-52.

24. Seeley WW, Menon V, Schatzberg AF, et al. Dissociable intrinsic connectivity networks for salience processing and executive control. J Neurosci 2007;27:2349-2356.

25. Zhou J, Greicius MD, Gennatas ED, et al. Divergent network connectivity changes in behavioural variant frontotemporal dementia and Alzheimer's disease. Brain 2010; 133:1352-1367.

26. Smith SM, Jenkinson M, Woolrich MW, et al. Advances in functional and structural MR image analysis and implementation as FSL. Neuroimage 2004;23(suppl 1): S208-S219.

27. Greicius MD, Krasnow B, Reiss AL, Menon V. Functional connectivity in the resting brain: a network analysis of the default mode hypothesis. Proc Natl Acad Sci USA 2003; 100:253-258.

28. Margulies DS, Kelly AM, Uddin LQ, Biswal BB, Castellanos FX, Milham MP. Mapping the functional connectivity of anterior cingulate cortex. Neuroimage 2007; 37:579-588.

29. Verhage F. Intelligence and Age: Research on Dutch People Aged Twelve to Seventy-Seven Years Old [in Dutch]. Assen: Van Gorcum; 1964.

30. Torralva T, Roca M, Gleichgerrcht E, Bekinschtein T, Manes F. A neuropsychological battery to detect specific executive and social cognitive impairments in early frontotemporal dementia. Brain 2009;132:1299-1309.

31. Geschwind DH, Robidoux J, Alarcon M, et al. Dementia and neurodevelopmental predisposition: cognitive dysfunction in presymptomatic subjects precedes dementia by decades in frontotemporal dementia. Ann Neurol 2001;50:741-746.

32. Hornberger M, Geng J, Hodges JR. Convergent grey and white matter evidence of orbitofrontal cortex changes related to disinhibition in behavioural variant frontotemporal dementia. Brain 2011;134:2502-2512.

33. Haller S, Nguyen D, Rodriguez C, et al. Individual prediction of cognitive decline in mild cognitive impairment using support vector machine-based analysis of diffusion tensor imaging data. J Alzheimers Dis 2010; 22:315-327.

34. Song SK, Sun SW, Ju WK, Lin SJ, Cross AH, Neufeld AH. Diffusion tensor imaging detects and differentiates axon and myelin degeneration in mouse optic nerve after retinal ischemia. Neuroimage 2003;20:17141722.

35. Song SK, Sun SW, Ramsbottom MJ, Chang C, Russell J, Cross AH. Dysmyelination revealed through MRI as increased radial (but unchanged axial) diffusion of water. Neuroimage 2002;17:1429-1436. 
36. Rohrer JD, Ridgway GR, Modat M, et al. Distinct profiles of brain atrophy in frontotemporal lobar degeneration caused by progranulin and tau mutations. Neuroimage 2010;53:1070-1076.

37. Whitwell JL, Jack CR Jr, Boeve BF, et al. Voxel-based morphometry patterns of atrophy in FTLD with mutations in MAPT or PGRN. Neurology 2009;72:813-820.

38. Rombouts SA, van Swieten JC, Pijnenburg YA, Goekoop R, Barkhof F, Scheltens P. Loss of frontal
fMRI activation in early frontotemporal dementia compared to early AD. Neurology 2003;60:19041908.

39. Schmahmann JD, Sherman JC. The cerebellar cognitive affective syndrome. Brain 1998;121:561-579.

40. Damoiseaux JS, Greicius MD. Greater than the sum of its parts: a review of studies combining structural connectivity and resting-state functional connectivity. Brain Struct Funct 2009;213:525-533.

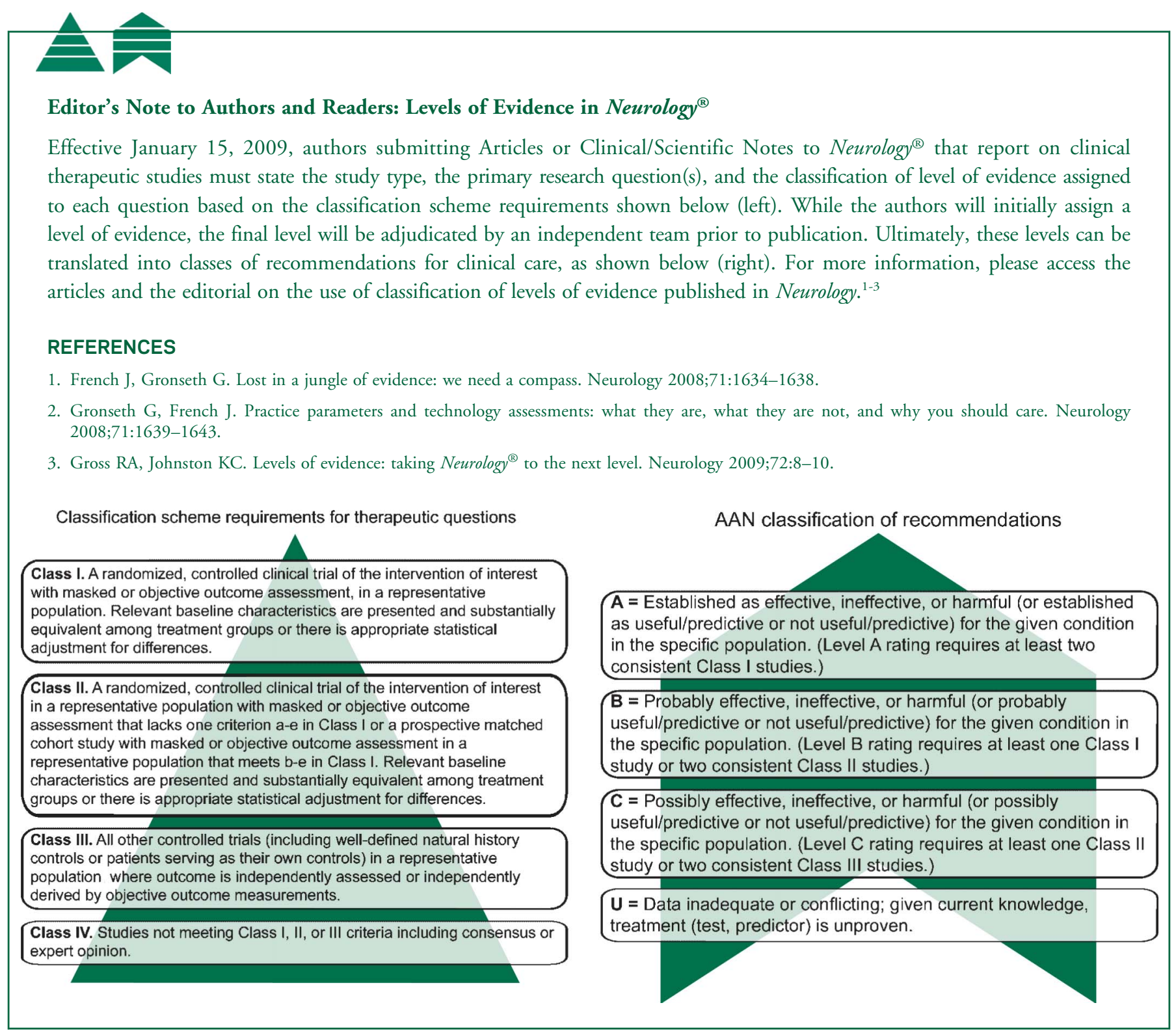

